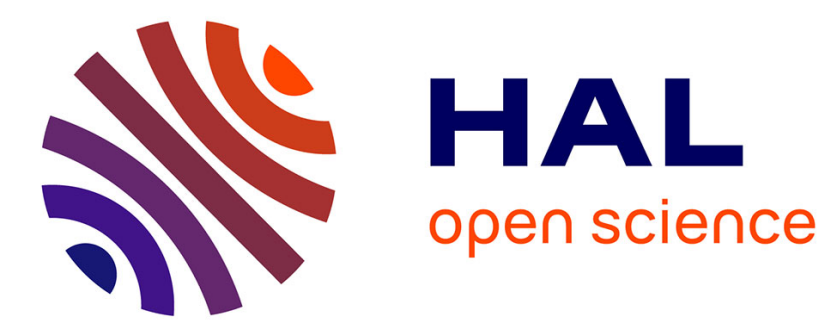

\title{
Interactions génétiques entre pathotypes français d'Heterodera avenae Woll. et variétés d'orge: I. - Aspect variétal
}

Françoise Person-Dedryver, Gérard Doussinault, Danielle Pannetier

\section{To cite this version:}

Françoise Person-Dedryver, Gérard Doussinault, Danielle Pannetier. Interactions génétiques entre pathotypes français d'Heterodera avenae Woll. et variétés d'orge: I. - Aspect variétal. Agronomie, 1984, 4 (8), pp.763-771. hal-00884694

\section{HAL Id: hal-00884694 \\ https://hal.science/hal-00884694}

Submitted on 1 Jan 1984

HAL is a multi-disciplinary open access archive for the deposit and dissemination of scientific research documents, whether they are published or not. The documents may come from teaching and research institutions in France or abroad, or from public or private research centers.
L'archive ouverte pluridisciplinaire HAL, est destinée au dépôt et à la diffusion de documents scientifiques de niveau recherche, publiés ou non, émanant des établissements d'enseignement et de recherche français ou étrangers, des laboratoires publics ou privés. 


\section{Interactions génétiques entre pathotypes français d'Heterodera avenae Woll. et variétés d'orge : I. - Aspect variétal}

Françoise PERSON-DEDRYVER \& Gérard DOUSSINAULT (*)

avec la collaboration technique de Danielle PANNETIER

I.N.R.A., Laboratoire de Zoologie, Station d'Amélioration des Plantes (*), Centre de Recherches de Rennes, B.P. 29. F 35650 Le Rheu

Une étude génétique du comportement vis-à-vis des 4 pathotypes français d'Heterodera avenae Woll. de 3 orges du test international des pathotypes de ce nématode : « Siri », " Ortolan ", « P 31-322-1 », et d'une orge française, "Vogue ", est entreprise pour différencier les gènes responsables de la virulence de ces pathotypes. Les pathotypes sont élevés dans des boîtes de Petri sur des plantes issues de croisements intervariétaux entre plantes résistantes ou résistante et multiplicatrice, sur agar.

Ce travail révèle la présence de nouveaux gènes dominants de résistance à $H$. avenae différents des gènes $H a$ et $\mathrm{Ha} 2$ déjà bien étudiés par ANDERSEN avec les 2 pathotypes danois Ha 11 (A) et $\mathrm{Ha} 12$ (C) :

- $\mathrm{AB}$, gènes complémentaires, et $\mathrm{C}$ dans « Siri », efficaces à l'encontre respectivement des pathotypes Fr 2 et Fr 3.

- EF et GH, 2 couples de gènes complémentaires dans "Ortolan », responsables de la résistance respectivement à $\mathrm{Fr} 1$ et $\mathrm{Fr} 3$.

- IJ et GL, 2 couples de gènes complémentaires dans « $\mathrm{P}$ 31-322-1 ", induisant la résistance respectivement à Fr 2 et Fr 3.

- EF, gènes complémentaires, et $P$ dans «Vogue », agissant sur, respectivement, les pathotypes Fr 1 et Fr 3. Fr 4 peut être considéré comme équivalent du pathotype danois $\mathrm{Ha} 12$ (C). Contrairement aux conclusions d'ANDERSEN, les pathotypes Fr 2 et $\mathrm{Fr} 4$, d'une part, et Fr 3 et $\mathrm{Ha} 11$ (A), d'autre part, s'avèrent différents puisque les systèmes génétiques mis en jeu pour les résistances ne sont pas les mêmes.

Mots clés additionnels : Nématode, gène, résistance, Hordeum.

In France 4 pathotypes of Heterodera avenae have been previously distinguished and called $\operatorname{Fr} 1, \operatorname{Fr} 2, \operatorname{Fr} 3$ and Fr 4. Their behaviour and that of the corresponding resistance genes have been studied in 4 barley lines: 'Siri', 'Ortolan', 'P 31-322-1' and 'Vogue'.

ANDERSEN, in Denmark, has studied the relationship between 3 of these lines and 2 pathotypes: Ha 11 (A) and $\mathrm{Ha} 12$ (C). Thus it has been of interest to compare the pathotypes described by ANDERSEN with the French ones and possibly to associate then.

The French pathotypes were bred on the barley lines, and on the F 1 and $F 2$ crosses between these lines. Young seedlings were grown in Petri dishes on agar and root tips were infected with larvae. The analysis of the development of females on the root surface allowed the behaviour of each plant to be tested.

New dominant genes for resistance to $H$. avenae were identified. These genes differed in particular from those described by ANDERSEN as Ha 1 and Ha 2.

- 'Siri' has 2 complementary genes AB effective against Fr 2 and a single dominant gene $C$ against Fr 3.

- 'Ortolan' has 2 complementary genes EF effective towards Fr 1 and also two complementary genes GH towards Fr 3.

- 'P 31-322-1' also carries 2 couples of complementary genes, $\mathrm{IJ}$ and GL, inducing resistance towards respectively Fr 2 and Fr 3.

- 'Vogue' has 2 complementary genes EF effective against Fr 1 and a single dominant gene P against Fr 3. Our results confirmed that $\mathrm{Fr} 4$ is similar to ANDERSEN's pathotype Ha 12. However, in contrast to ANDERSEN's results we showed that the Fr 2 and Fr 4 pathotypes really differ from each other as do Fr 3 and Ha 11 since the genetic systems involved in the resistance were found different in both cases.

Additional key words : Nematode, gene, resistance, Hordeum. 


\section{INTRODUCTION}

Les 4 pathotypes français, Fr 1, Fr 2, Fr 3 et Fr 4 d'Heterodera avenae Woll. ont été différenciés par leur capacité à se multiplier ou non sur différentes variétés d'orge ou d'avoine (RIVOAL, 1977). Cet auteur a montré que 2 pathotypes septentrionaux, Fr 3 et Fr 4, sont similaires, respectivement, aux pathotypes $\mathrm{A}$ et $\mathrm{C}$ trouvés en Europe du Nord, et plus particulièrement en Hollande, Grande-Bretagne, Suède et Danemark, alors que Fr 1 et Fr 2 n'ont apparemment pas d'équivalents en Europe. Ces résultats sont repris par ANDERSEN \& ANDERSEN (1982), lorsqu'ils proposent d'établir une terminologie commune concernant tous les pathotypes d' $H$. avenae trouvés de par le monde. Si ANDERSEN prend en compte 3 d'entre eux, qu'il appelle $\mathrm{Ha} 41$ pour Fr 1, Ha 11 pour Fr 3 et $\mathrm{A}, \mathrm{Ha} 12$ pour Fr 4 et $\mathrm{C}$; par contre, il confond $\mathrm{Fr} 4$ et Fr 2 en un seul et même pathotype. En effet, Fr 2 et Fr 4 provoquent des réponses identiques, lorsqu'ils sont confrontés aux variétés d'orge, de blé et d'avoine reconnues comme hôtes différentiels sur le plan international. Ces pathotypes diffèrent cependant par leur capacité à se multiplier sur d'autres cultivars d'avoines, tels que « Noire de Moyencourt " (RIVOAL \& PERSON-DEDRYVER, 1982).

En considérant tous ces résultats, nous voyons qu'en France, nous sommes confrontés à des situations, en ce qui concerne l'emploi des cultivars résistants, différentes de celles enregistrées dans les pays du Nord de l'Europe, puisque nous avons 2 pathotypes Fr 1 et Fr 2 différents de ceux trouvés à l'étranger. L'origine des variétés d'orge résistantes à $H$. avenae étant le plus souvent britannique, suédoise ou danoise, il s'avère nécessaire de connaître avec exactitude la réponse des géniteurs et des variétés utilisées et le mode d'hérédité de leur résistance, à l'encontre des 4 pathotypes rencontrés en France.

Le travail entrepris concerne l'étude génétique de la résistance de 4 variétés d'orge choisies pour permettre l'étude de gènes de virulence des pathotypes français. L'élevage des nématodes sur les plantes issues de divers croisements intervariétaux a été réalisé en conditions contrôlées dans des boîtes de Petri, suivant la méthode décrite par PERSON \& DOUSSinaulT (1978). Cette technique permet une étude génétique du comportement des différents génotypes des orges cultivées.

\section{MATÉRIEL ET MÉTHODES}

\section{A. Le nématode}

Les 4 pathotypes français d' $H$. avenae utilisés dans cette étude, et définis par RIVOAL (1977), sont d'origines géographiques et climatiques très différentes. Deux d'entre eux proviennent des zones septentrionales: Fr 3 et Fr 4, respectivement des départements de l'Orne (Argentan) et de la Marne (Nuisement). Fr 2 est trouvé plus au sud, dans le département de la Vienne (Vivonne). Fr 1 est originaire du département de l'Aude (Villasavary). Les larves infestantes L 2 du nématode utilisées sont issues de kystes provenant de sol ayant porté une culture hôte, elles sont mises dans de l'eau 2 mois à $20^{\circ} \mathrm{C}$ puis à $5{ }^{\circ} \mathrm{C}$ afin de lever leur diapause (RIVOAL, 1979).

\section{B. Les variétés d'orge}

Nombreuses sont les variétés qui présentent des gènes de résistance à l'encontre d' $H$. avenae, bloquant le développement des femelles du parasite et donc sa multiplication. Nous avons choisi d'étudier plus particulièrement 4 d'entre elles : "Siri », "Ortolan » «P 31322-1" et "Vogue ", pour les raisons suivantes: " Ortolan » et "Siri » appartiennent à la gamme des cultivars des hôtes différentiels pour les pathotypes d' $H$. avenae et leur résistance dépend pour chacune d'entre elles d'un gène dominant connu, $\mathrm{Ha} 1$ et $\mathrm{Ha} 2$. Ces 2 gènes $\mathrm{Ha} 1$ et $\mathrm{Ha} 2$ sont indépendants et responsables respectivement de la résistance de "Siri " à l'encontre des 2 pathotypes danois, $\mathrm{Ha} 11$ (A) et $\mathrm{Ha} 12$ (C), et de la résistance d' "Ortolan » vis-à-vis du pathotype HA 11 (ANDERSEN \& ANDERSEN, 1982). " Siri » est résistante à l'ensemble de 3 de nos pathotypes : Fr 2, Fr 3 et Fr 4, mais permet la multiplication de Fr 1 (tabl. 1). "Ortolan » n'est résistant qu'à Fr 1 et Fr 3, de même que "Vogue », variété française inscrite au catalogue jusqu'en 1981. La résistance de "Vogue » n'étant pas étudiée par ailleurs, il nous semble intéressant de voir si elle dépend du même système génique que «Ortolan ".

«P 31-322-1 » est issue de la sélection à partir d'un croisement entre plantes possédant chacune un des 2 gènes de résistance à $H$. avenae, $\mathrm{Ha} 1$ et $\mathrm{Ha} 2$, et est

TABLEAU

Comportement de diverses variétés d'orge à l'encontre des pathotypes d'Heterodera avenae.

The behaviour and the resistance genes involved in 4 barley lines against the pathotypes of Heterodera avenae.

\begin{tabular}{|c|c|c|c|c|c|c|c|}
\hline & \multirow{2}{*}{$\begin{array}{c}\text { Pathotypes } \\
\text { d'Heterodera avenae }\end{array}$} & \multicolumn{2}{|c|}{ Danois } & \multicolumn{4}{|c|}{ Français } \\
\hline & & Ha 11 (A) & $\mathrm{Ha} 12(\mathrm{C})$ & Fr 1 & Fr 2 & Fr 3 & Fr 4 \\
\hline & Variétés d'orge et origine & \multicolumn{2}{|c|}{$\begin{array}{c}\text { gène de résistance à } H \text {. avenae } \\
\text { ANDERSEN } 1975\end{array}$} & & & & \\
\hline Aramir & inscrites au catalogue & - & - & $M$ & M & $M$ & M \\
\hline Vogue & of ficiel français & - & - & $\mathbf{R}$ & $\mathbf{M}$ & $\mathrm{R}$ & M \\
\hline Ortolan & variétés du test international & Ha 1 & - & $\mathbf{R}$ & $\mathbf{M}$ & $\mathrm{R}$ & $\mathbf{M}$ \\
\hline Siri & des pathotypes d' $H$. avenae & Ha 2 & $\mathrm{Ha} 2$ & M & $\mathrm{R}$ & $\mathrm{R}$ & $\mathbf{R}$ \\
\hline \multicolumn{2}{|c|}{ P $31-322-1$} & $\mathrm{Ha} 1$ et $\mathrm{Ha} 2$ & Ha 2 & M & $\mathbf{R}$ & $\mathrm{R}$ & $\mathbf{R}$ \\
\hline
\end{tabular}

M : multiplicateur ; $\mathbf{R}$ : résistant. 
résistant aux pathotypes danois $\mathrm{Ha} 11$ et $\mathrm{Ha} 12$ (ANDERSEN, 1975). Si les 2 gènes $\mathrm{Ha} 1$ et $\mathrm{Ha} 2$ sont les seuls gènes responsables de la résistance des 2 variétés «Siri » et «Ortolan » à l'égard des pathotypes français d' $H$. avenae, comme ils le sont vis-à-vis des 2 pathotypes danois, leur présence dans «P 31-322-1 » devrait induire la résistance de cette variété aux 4 pathotypes Fr 1, Fr 2, Fr 3 et Fr 4 . Or le pathotype Fr 1 se multiplie sur «P 31-322-1» (tabl. 1). Une étude génétique de la résistance de « Siri », « P 31-3221 » et «Ortolan » vis-à-vis de Fr 1, ainsi que des autres pathotypes français, devrait permettre de comprendre la nature de ces génotypes.

La variété « Aramir », inscrite au catalogue français, multiplicatrice des 4 pathotypes d' $H$. avenae étudiés, servira comme parent multiplicateur dans les croisements intervariétaux.

\section{Les méthodes}

Diverses méthodes sont à notre disposition pour réaliser l'élevage des nématodes sur céréales en conditions, soit extérieures sur sol contaminé, soit contrôlées en boîtes de Petri (RIVOAL et al., 1978). Nous avons opté pour la méthode utilisant la culture des céréales sur agar agar coulé en boîtes de Petri (PERSON \& DoUSSINAULT, 1978), car elle est plus adaptée aux contraintes de notre étude : un grand nombre de plantes à observer individuellement sur un court laps de temps, 2 mois après la mise en culture. Cette méthode permet en outre la réalisation des tests dans des conditions ambiantes constantes et des observations très aisées. Les plantes sont ensuite mises en terre et effectuent leur cycle végétatif normal, permettant l'analyse de la génération suivante.

Des croisements ont été réalisés entre la variété multiplicatrice «Aramir » et chacune des 4 variétés résistantes. Le comportement des 4 pathotypes français d' $H$. avenae a été noté sur 10 plantes $\mathrm{F} 1$ et une cinquantaine de plantes $\mathrm{F} 2$ issues de ces croisements. Pour vérifier l'identité des gènes de résistance, nous avons réalisé des croisements entre lignées résistantes : « 31-322-1 » $\times$ «Vogue », «Ortolan » $\times$ « Siri »,
«Vogue » $x$ «Siri ", «Siri » $x$ «Ortolan ». La sélection dans ces croisements pourrait être à l'origine de génotypes résitants à l'encontre des 4 pathotypes d'H. avenae en France.

\section{RÉSULTATS}

\section{A. Détermination des plantes multiplicatrices et résis- tantes parmi les descendants $F 2$ d'un croisement intervariétal}

Les conditions d'utilisation de la méthode d'élevage d'H. avenae sur plantes cultivées dans les conditions contrôlées des boîtes de Petri ont été mises au point pour permettre de détecter sans ambiguïté, sur 10 répétitions de 10 plantes, un cultivar résistant d'un cultivar multiplicateur (PERSON \& DOUSSINAULT, 1978). Il est possible de trouver sur les 10 répétitions d'un cultivar résistant une seule plante qui permette la formation de femelles avec œufs en nombre restreint. A l'inverse, sur variété multiplicatrice, il est possible, dans de très rares cas, d'avoir 1 plante sur les 10 qui ne présente aucune femelle, sans doute pour des raisons de défaut d'infestation. Au cours de ce travail, nous avons trouvé des plantes avec 1 ou 2 femelles sans œufs. Il s'agit, soit de plantes résistantes qui ne permettent pas la reproduction des femelles, soit de plantes multiplicatrices, non infestées. Dans une étude génétique de la résistance, où il faut connaître avec précision le génotype de chaque plante, il se pose le problème de la classification des plantes de ce type. Pour l'analyse des données recueillies en F 2, nous avons tranché, en cas d'ambiguïté, en comparant les résultats enregistrés à ceux obtenus avec les parents multiplicateurs, résistants, et leurs descendants F 1 .

Nous citons ici 2 exemples obtenus après l'étude de 2 croisements d'orge "Aramir" $x$ "Vogue 》 et "Aramir » $x$ 《 Ortolan » à l'encontre respectivement des pathotypes Fr 1 et Fr 3 d'H. avenae.

Pour le $1^{\text {er }}$ croisement (tabl. 2), toutes les plantes du parent multiplicateur «Aramir » présentent une ou plusieurs femelles avec œufs, ce qui prouve les bonnes

TABLEAU 2

Répartition des plantes multiplicatrices ou résistantes à l'encontre du pathotype Fr 1 d'Heterodera avenae dans les descendants F I et F 2 issus du croisement entre variétés d'orge " Aramir 》 et « Vogue ".

Frequency distribution of plants resistant or susceptible to the Fr 1 pathotype of Heterodera avenae in the $F I$ and $F 2$ lines resulting from the cross between 2 barley varieties 'Aramir' and 'Vogue'.

\begin{tabular}{|c|c|c|c|c|}
\hline Effectif de plantes avec: & $\begin{array}{c}\text { Parent multiplicateur } \\
\text { Aramir }\end{array}$ & $\begin{array}{l}\text { Parent resistant } \\
\text { Vogue }\end{array}$ & $\begin{array}{l}\text { Descendants } \\
\quad \text { F } 1\end{array}$ & $\begin{array}{l}\text { Descendants } \\
\quad \text { F } 2\end{array}$ \\
\hline $\begin{array}{l}\text { - } 0 \text { femelle } \\
\text { - femelles vides }\end{array}$ & & $10 \mathrm{R}$ & $\left.{ }_{2}^{8}\right\} R$ & $\left.\begin{array}{c}29 \\
5\end{array}\right\} \mathrm{R}$ \\
\hline $\begin{array}{l}\text { - } 1 \text { femelle avec œufs } \\
+ \text { éventuellement } \\
\text { femelles vides } \\
\text { _ de } 2 \text { à } 11 \text { femelles avec œufs }\end{array}$ & ${ }_{38}^{2} \mathrm{M}$ & & & ${ }_{15}^{9} \mathrm{M}_{\longrightarrow 2 \mathrm{M}}$ \\
\hline Total & 40 & 10 & 10 & 58 \\
\hline
\end{tabular}

M : multiplicateur ; $R$ : résistant. 
TABLEAU 3

Répartition des plantes multiplicatrices ou résistantes à l'encontre du pathotype Fr $3 d$ 'Heterodera avenae dans les descendants $F 1$ et $F 2$ issus du croisement entre variétés d'orge "Aramir " et "Ortolan ».

Frequency distribution of plants resistant or susceptible to the Fr 3 pathotype of Heterodera avenae in the $F I$ and $F 2$ lines resulting from the cross between 2 barley varieties 'Aramir' and 'Ortolan'.

\begin{tabular}{|c|c|c|c|c|}
\hline Effectif de plantes avec: & $\begin{array}{c}\text { Parent multiplicateur } \\
\text { «Aramir » }\end{array}$ & $\begin{array}{c}\text { Parent resistant } \\
\text { «Ortolan } »\end{array}$ & $\begin{array}{l}\text { Descendants } \\
\text { F } 1\end{array}$ & $\begin{array}{l}\text { Descendants } \\
\text { F } 2\end{array}$ \\
\hline - 0 femelle & $1\} \begin{array}{l}\text { environ } \\
5 \% \text { des }\end{array}$ & $8\} R$ & $7\} R$ & $17\left\{\begin{array}{l}100 \% \text { des } \mathrm{R} \\
+ \text { environ }\end{array}\right.$ \\
\hline — femelles vides & 1) M & $2)$ & $4 J$ & 16 $5 \%$ des $M$ \\
\hline $\begin{array}{c}\text { - } 1 \text { femelle avec aufs } \\
+ \text { éventuellement } \\
\text { femelles vides }\end{array}$ & $15\} \begin{array}{l}\text { environ } \\
95 \% \text { des }\end{array}$ & & & $4\left\{\begin{array}{l}\text { environ } \\
95 \% \text { des }\end{array}\right.$ \\
\hline - de 2 à 10 femelles avec ceufs & $20 \int \mathrm{M}$ & & & $16 \mathrm{M}$ \\
\hline Total & 37 & 10 & 11 & 53 \\
\hline
\end{tabular}

$\mathrm{M}$ : multiplicateur ; $\mathrm{R}$ : résistant.

conditions d'utilisation du test. Nous avons donc considéré, pour les générations $\mathrm{F} 1$ et $\mathrm{F} 2$ issues de ce croisement, que toute plante sans femelle ou avec femelle vide est résistante, et nous obtenons ainsi, en $\mathrm{F} 1$, 10 plantes résistantes et, en $\mathrm{F} 2,34$ plantes résistantes et 24 plantes multiplicatrices.

Pour le $2^{\mathrm{e}}$ croisement (tabl. 3), environ 5 p. 100 des plantes du parent multiplicateur "Aramir » sont sans femelle ou avec femelles vides et peuvent être confondues avec des plantes du parent résistant «Ortolan». La F 1, homogène phénotypiquement, est considérée résistante, puisqu'aucune plante ne présente de femelle avec œufs. En F 2, nous considérons, à l'image du témoin multiplicateur, que seules 95 p. 100 des plantes multiplicatrices sont traduites par les plantes ayant une ou plusieurs femelles avec œufs et que les 5 p. 100 autres sont confondues avec les plantes résistantes sans femelle ou avec femelles vides. Nous estimons donc en F 2 que 21 plantes sont multiplicatrices et 32 résistantes.

\section{B. Croisements entre parent multiplicateur "Aramir » et variétés présentant une résistance à l'encontre d'H. avenae}

L'analyse des plantes F 1 montre que les gènes de résistance trouvés dans les 4 cultivars résistants sont dominants en ce qui concerne 3 des pathotypes français d' $H$. avenae, Fr 1, Fr 3 et Fr 4.

La formation de 1 ou au maximum 2 femelles fécondes Fr 2 sur quelques plantes $F 1$ issues des croisements entre le parent multiplicateur «Aramir » et les orges « Siri » ou «P 31-322-1 » prouve que les résistances de "Siri » et «P 31-322-1 » dépendent de gènes incomplètement dominants en ce qui concerne le pathotype Fr 2.

Les résultats obtenus en $\mathrm{F} 2$ sont plus complexes et diffèrent suivant le cultivar et le pathotype considérés (tabl. 4). A chaque gène trouvé, nous affecterons une lettre de l'alphabet. Dans une partie ultérieure de cette étude, nous essaierons de voir s'il est possible de les identifier entre eux et aux gènes de résistance déjà connus et cités dans la bibliographie.
Les ségrégations observées sont en accord avec les hypothèses suivantes :

- «Siri »: La résistance serait induite par 2 gènes dominants, $C$ et $D$, vis-à-vis respectivement des pathotypes Fr 3 et Fr 4 et par 2 gènes dominants complémentaires, A et B, à l'encontre du pathotype Fr 2 . Actuellement, un seul gène dominant, $\mathrm{Ha} 2$, est connu dans ce cultivar (ANDERSEN, 1975).

- «Ortolan »: 2 gènes dominants complémentaires, EF et $\mathrm{GH}$, seraient mis en évidence en ce qui concerne respectivement nos 2 pathotypes Fr 1 ou Fr 3. Par contre, ANDERSEN ne cite qu'un gène dominant de résistance dans le cultivar «Ortolan »: Ha 1.

- «P 31-322-I 》:

1) 1 gène dominant, $M$, serait responsable de sa résistance à l'encontre du pathotype Fr 4 .

2) 2 gènes dominants complémentaires, $\mathrm{KL}$, induiraient sa résistance au pathotype Fr 3 .

3) la résistance vis-à-vis de Fr 2 dépendrait de 2 gènes dominants complémentaires, IJ.

Ce cultivar ne présente, d'après ANDERSEN (1975), que 2 gènes dominants de résistance vis-à-vis d' $H$. avenae, $\mathrm{Ha} 1$ et $\mathrm{Ha} 2$.

- "Vogue »: 2 gènes dominants complémentaires, NO, seraient responsables de sa résistance vis-à-vis de Fr 1 , mais 1 seul gène dominant, $P$, permet d'expliquer le comportement à l'encontre de Fr 3.

\section{Croisements entre variétés d'orge présentant une résistance à $H$. avenae}

Nous avons jugé qu'il n'était pas nécessaire de confirmer les résultats trouvés en ce qui concerne la résistance de «Siri » et de «P 31-322-1 » vis-à-vis du pathotype Fr 4 puisqu'ils sont conformes à ceux décrits par ANDERSEN \& ANDERSEN (1970) avec le pathotype Ha 12 (C) similaire à $\mathrm{Fr} 4$. Nous considérons que c'est le gène $\mathrm{Ha} 2$ qui agit dans ces 2 cultivars à l'égard de Fr 4 (Ha $2=\mathrm{D}=\mathrm{M}$ ).

Par contre, nous tenions à préciser ceux enregistrés après étude de la résistance des 4 cultivars à l'égard des 3 autres pathotypes d' $H$. avenae, Fr 1, Fr 2 et $\operatorname{Fr} 3$, 
TABLEAU 4

Etude du mode d'hérédité de la résistance de 4 cultivars d'orge à Heterodera avenae en France : analyse de la descendance $F 2$. Inheritance of the resistance of 4 barley varieties to Heterodera avenae in France : study of the F 2 progeny.

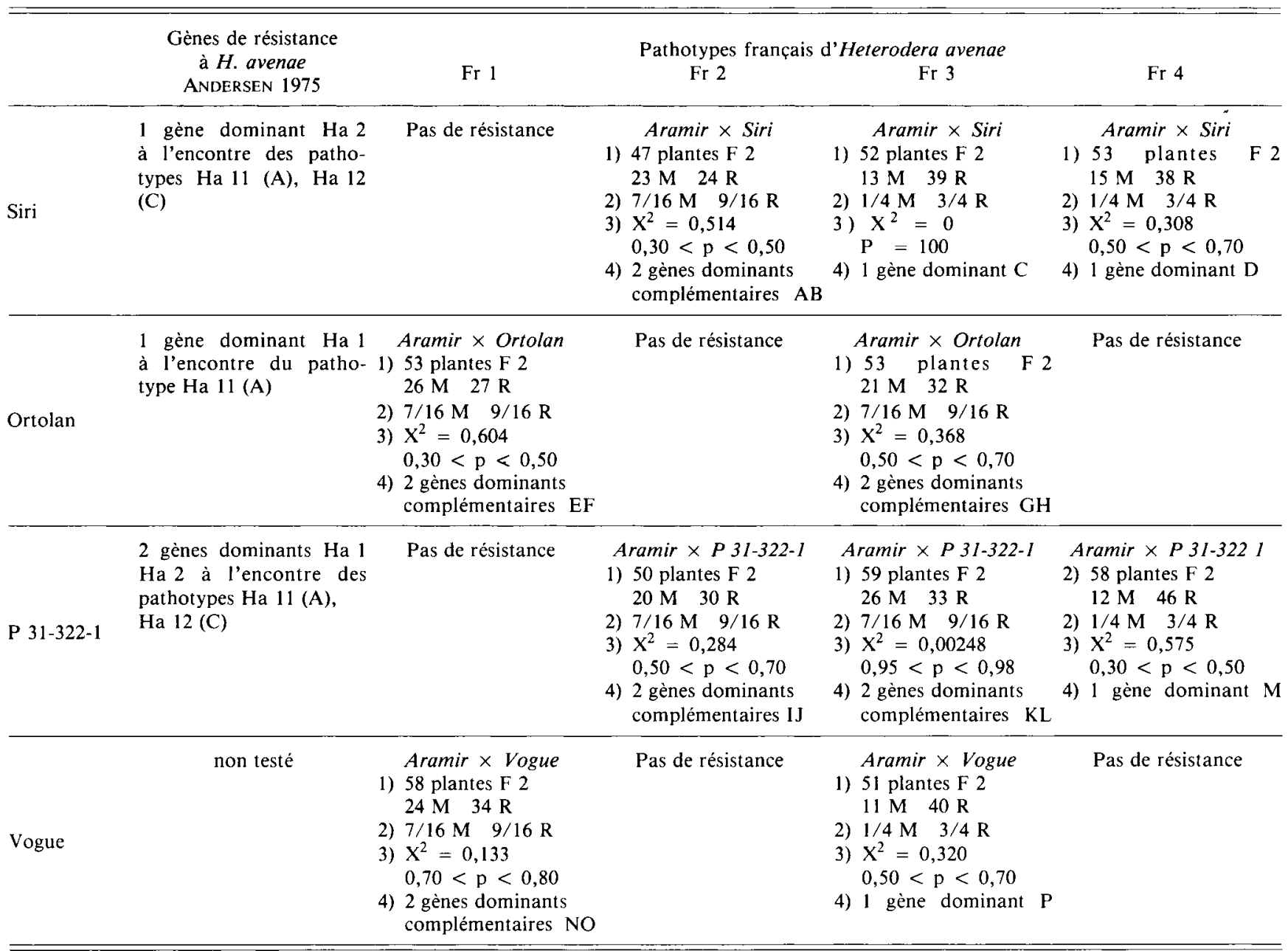

$\mathrm{R}$ : résistant ; $M$ : multiplicateur ; 1) résultats ; 2) hypothèse ; 3) test de l'hypothèse ; 4) conclusion.

puisque nous trouvons des différences avec les résultats cités en bibliographie. Pour cela, nous avons réalisé divers croisements entre cultivars d'orge présentant une résistance à $H$. avenae. En F 1, nous trouvons, comme précédemment, le caractère dominant des résistances. L'analyse de la F 2 étant plus complexe, nous commenterons successivement les résultats trouvés par pathotype.

\section{Pathotype Fr 1 (tabl. 5)}

- l'étude des croisements « Siri » $x$ « Ortolan » et «Ortolan » $x$ 《 P 31-322-1 » confirme la présence de 2 gènes de résistance dominants complémentaires, $E$ et F, dans le cultivar "Ortolan ». Nous démontrons en même temps que ces 2 gènes sont indépendants de $\mathrm{Ha} 2$ $(=\mathrm{D}=\mathrm{M}), \mathrm{AB}, \mathrm{C}$, IJ et $\mathrm{KL}$;

- le comportement vis-à-vis de Fr 1 des diverses plantes F 2 issues des croisements «Vogue " $\times$ «Siri » et «P 31-322-1 » $x$ " Vogue » confirme la présence de 2 gènes de résistance dominants et complémentaires, NO dans la variété "Vogue ", qui sont eux aussi indépendants de $\mathrm{Ha} 2, \mathrm{AB}, \mathrm{C}, \mathrm{IJ}$ et $\mathrm{KL}$;

- la similitude des résultats obtenus après analyse de ces 2 croisements nous laisse penser que les gènes
$\mathrm{EF}$, agissant dans "Ortolan », sont identiques à ceux rencontrés dans "Vogue ». Les plantes F 2 issues du croisement « Ortolan » $x$ " Vogue » sont toutes résistantes à $F r 1$ et démontrent qu'effectivement les gènes agissant dans les 2 cultivars sont les mêmes $(E F=N O)$.

\section{Pathotype Fr 2 (tabl. 6)}

- l'analyse des plantes $F 2$ issues des 2 croisements "Siri » $x$ «Ortolan » et "Vogue " $x$ «Siri " prouve une fois de plus l'action complémentaire de 2 gènes de résistance dominants, $\mathrm{AB}$, dans le cultivar «Siri ». Ces 2 gènes sont indépendants de EF $(=\mathrm{NO})$, $\mathrm{GH}$ et $\mathrm{P}$;

- de la même façon, après réalisation des croisements «Ortolan » $x$ 《 $\mathrm{P}$ 31-322-1 " et « P 31-322-1» $X$ "Vogue », nous trouvons 2 gènes de résistance dominants complémentaires, I et $\mathbf{J}$, agissant dans « $\mathrm{P}$ 31-322-1 ». Ces 2 gènes, I et $\mathrm{J}$, sont eux aussi indépendants des gènes $\mathrm{EF}(=\mathrm{NO})$; $\mathrm{GH}$ et $\mathrm{P}$;

- les résultats enregistrés après étude de ces 2 types de croisements nous font penser à une identité de gènes IJ et AB dans les cultivars "Siri » et «P 31-322-1". Nous ne pouvons le vérifier actuellement, car nous 


\section{TABLEAU 5}

Etude du mode d'hérédité de la résistance de 4 cultivars d'orge au pathotype Fr I d'Heterodera avenae : analyse de la descendance $F 2$. Inheritance of the resistance of four barley varieties to patholvpe Fr 1 of Heterodera avenae : study of the F 2 progeny.

\begin{tabular}{|c|c|c|}
\hline $\begin{array}{l}\text { Croisement "Siri " } \times \text { "Ortolan " } \\
\text { genes de resistance }\end{array}$ & $\begin{array}{l}\text { Croisement «Vogue » } \times \text { «Siri » } \\
\text { genes de resistance }\end{array}$ & $\begin{array}{l}\text { Croisement «Ortolan » } \times \text { «Vogue » } \\
\text { genes de resistance }\end{array}$ \\
\hline $\begin{array}{l}\text { Siri }=\text { multiplicateur de } \operatorname{Fr} 1 ; \mathrm{AB}(\mathrm{Fr} 2), \mathrm{C} \\
\quad(\operatorname{Fr} 3), \mathrm{Ha} 2=\mathrm{M}=\mathrm{D}(\mathrm{Fr} 4)\end{array}$ & $\begin{array}{l}\text { Siri }=\text { multiplicateur de Fr } 1 ; A B(\operatorname{Fr} 2), C \\
\quad(\operatorname{Fr} 3), \mathrm{Ha} 2=M=D(\operatorname{Fr} 4)\end{array}$ & $\begin{array}{l}\text { Ortolan }=\text { résistant à Fr } 1, \mathrm{EF}(\mathrm{Fr} 1), \\
\mathrm{GH}(\mathrm{Fr} 3)\end{array}$ \\
\hline $\begin{array}{l}\text { Ortolan = résistant à Fr } 1 ; \mathrm{EF}(\mathrm{Fr} 1), \mathrm{GH} \\
(\text { Fr 3) }\end{array}$ & $\begin{array}{l}\text { Vogue = résistant à Fr } 1 ; \text { NO (Fr 1) } \\
\quad P(F r 3)\end{array}$ & $\begin{array}{l}\text { Vogue }=\text { résistant à Fr } 1, \text { NO (Fr } 1) \text {, } \\
\quad P(F r 3)\end{array}$ \\
\hline 1) 29 plantes $\mathrm{F} 2$ & 1) 27 plantes F 2 & 1) 121 plantes $\mathbf{F} 2$ \\
\hline $14 \mathrm{M} \quad 15 \mathrm{R}$ & $15 \mathrm{M} \quad 12 \mathrm{R}$ & $121 \mathrm{R}$ \\
\hline 2) $7 / 16 \mathrm{M} \quad 9 / 16 \mathrm{R}$ & 2) $7 / 16 \mathrm{M} \quad 9 / 16 \mathrm{R}$ & 4) 2 gènes dominants complémentai- \\
\hline 3) $X^{2}=0,240$ & 3) $\mathrm{X}^{2}=1.52$ & res identiques dans Ortolan et Vogue \\
\hline $0,50<p<0,70$ & $0,20<\mathrm{p}<0,30$ & $\mathrm{EF}=\mathrm{NO}$ \\
\hline 4) 2 gènes dominants complémentaires EF & 4) 2 gènes dominants complémentaires NO & \\
\hline EF indépendants de $\mathrm{AB}, \mathrm{C}, \mathrm{Ha} 2=\mathrm{D}=\mathrm{M}$ & NO indépendants de $\mathrm{AB}, \mathrm{C}, \mathrm{Ha} 2=\mathrm{M}=\mathrm{D}$ & \\
\hline $\begin{array}{l}\text { Croisement « Ortolan » } \times \ll \text { P 31-322-1 } \\
\text { genes de resistance }\end{array}$ & $\begin{array}{l}\text { Croisement «P 31-322-1 » } \times \text { «Vogue » } \\
\text { gènes de résistance }\end{array}$ & \\
\hline \multicolumn{3}{|l|}{ Ortolan = résistant à $\mathrm{Fr} 1 ; \mathrm{EF}(\mathrm{Fr} 1), \mathrm{GH}$} \\
\hline P $31-322-1=$ multiplicateur de Fr 1 & IJ $(\operatorname{Fr} 2), \mathrm{KL}(\operatorname{Fr} 3), \mathrm{Ha} 2=\mathrm{M}(\operatorname{Fr} 4)$ & $\mathbf{M}=$ multiplicateur \\
\hline IJ $($ Fr 2), KL (Fr 3), Ha $2=M(F r ~ 4)$ & Vogue $=$ résistant à Fr $1 ; \mathrm{NO}($ Fr 1), $\mathrm{P}(\mathrm{Fr} 3)$ & $\begin{array}{l}\text { 1) résultats } \\
\text { 2) hypothèse }\end{array}$ \\
\hline 1) 48 plantes $F 2$ & 1) 52 plantes $F 2$ & 3) test de l'hypothèse \\
\hline $17 \mathrm{M} 31 \mathrm{R}$ & $26 \mathrm{M} \quad 25 \mathrm{R}$ & 4) conclusion \\
\hline 2) $7 / 16 \mathrm{M} 9 / 16 \mathrm{R}$ & 2) $7 / 16 \mathrm{M} 9 / 16 \mathrm{R}$ & \\
\hline 3) $X^{2}=1,354$ & 3) $\mathrm{X}^{2}=1,085$ & \\
\hline $0,20<\mathrm{p}<0,30$ & $0,20<p<0,30$ & \\
\hline 4) 2 gènes dominants complémentaires EF & 4) 2 gènes dominants complémentaires NO & \\
\hline EF indépendants de $\mathrm{IJ}, \mathrm{KL}, \mathrm{Ha} 2=\mathrm{M}$ & NO indépendants de $\mathbf{I J}, \mathrm{KL}, \mathrm{Ha} 2=\mathbf{M}$ & \\
\hline
\end{tabular}

\section{TABLEAU 6}

Etude du mode d'hérédité de la résistance de 4 cultivars d'orge au pathotype Fr 2 d'Heterodera avenae en France : analyse de la descendance $F 2$. Inheritance of the resistance of four barley varieties to pathotype Fr 2 of Heterodera avenae: study of the F 2 progeny.

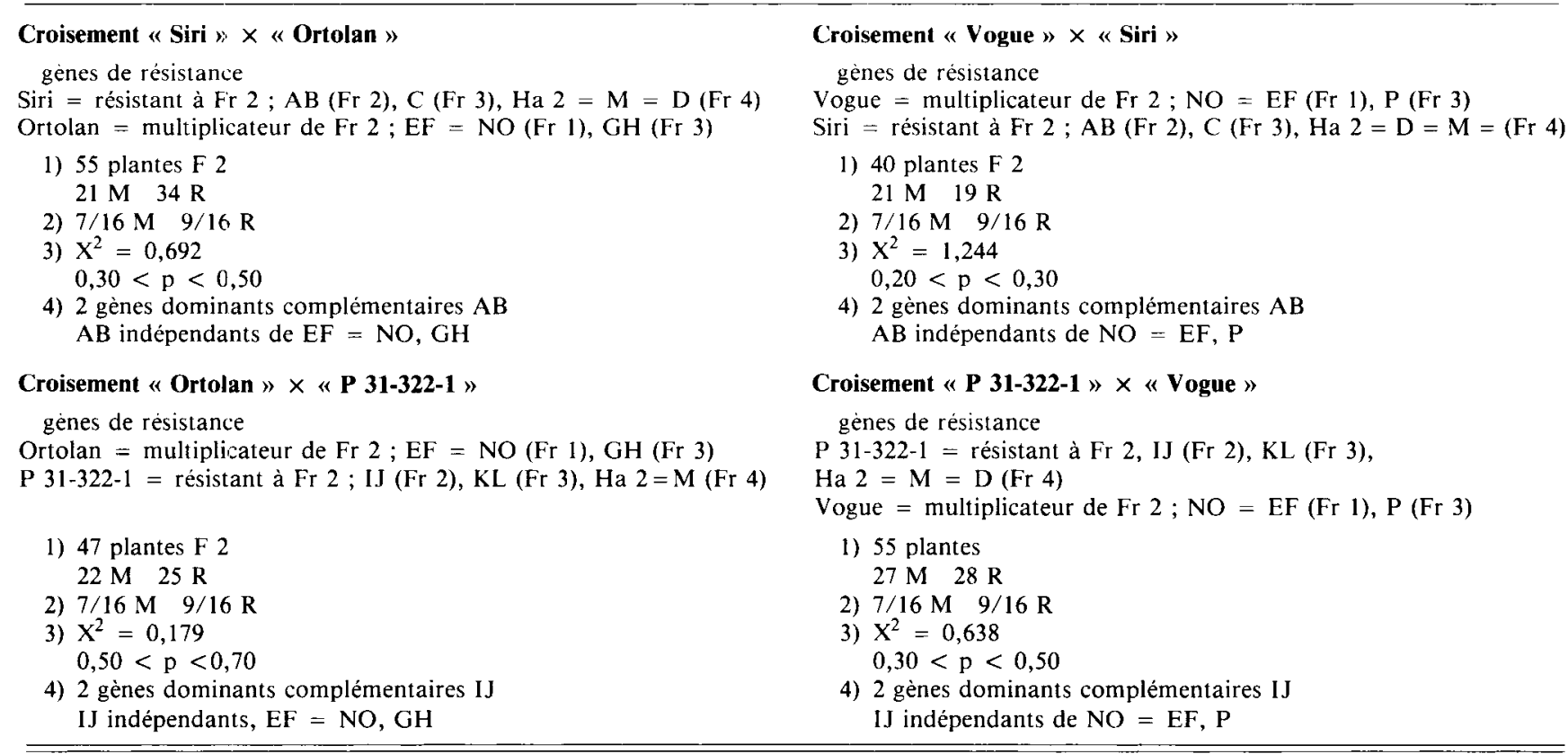

$\mathrm{R}$ : résistant ; $\mathrm{M}$ : multiplicateur ; 1) résultats ; 2) hypothèse ; 3) test de l'hypothèse ; 4) conclusion. 
n'avons pas de plantes $F 2$ issues de croisements entre ces 2 cultivars.

\section{Pathotype Fr 3 (tabl. 7)}

- l'étude des croisements «Siri » $x$ «Ortolan», "Ortolan » $x$ 《P 31-322-1 » et "Vogue » $x$ " Siri » est en accord avec les hypothèses suivantes :

1) 2 gènes dominants complémentaires, KL dans «P 31-322-1 » et GH dans «Ortolan », l'un des 2 étant commun dans les 2 cas $(K=G)$. Nous avons donc $\mathrm{K}(=\mathrm{G}) \mathrm{L}$ agissant dans « $\mathrm{P}$ 31-322-1 » et $\mathrm{G}$ (= K) $\mathrm{H}$ dans «Ortolan » ;

2) 1 gène dominant, $C$, dans «Siri », indépendant de $\mathrm{G}(=\mathrm{K}) \mathrm{H}, \mathrm{EF}, \mathrm{P}$ et NO (= EF). D'après l'étude des croisements «Aramir » $X$ «Siri » et «Aramir» $X$ «P 31-322-1», nous constatons que le gène $C$ ne peut être identifié à $\mathrm{Ha} 2$ en ce qui concerne Fr 3 , car ce gène $\mathrm{Ha} \mathrm{2}$, présent dans « $\mathrm{P}$ 31-322-1 », ne peut agir seul à l'encontre de ce pathotype ;

- l'effectif des plantes F 2 du croisement «P 31 322-1 " par "Vogue » est faible pour conclure avec sécurité dans le cas où 3 gènes sont impliqués. Mais les résultats obtenus en F 2 après croisement de «Vogue » par «Siri » et de «P 31-322-1 » par «Vogue», n'infirment pas l'existence d'un gène de résistance dominant, $P$, dans "Vogue », différent des gènes $G$ $(=\mathrm{K})$ et $\mathrm{H}$ d'« Ortolan », et indépendant de $\mathrm{Ha} 2$, $\mathrm{AB}, \mathrm{C}$, IJ et $\mathrm{G}(=\mathrm{K}) \mathrm{L}$.

\section{DISCUSSION ET CONCLUSIONS}

La méthode d'élevage d' $H$. avenae dans les conditions contrôlées des boîtes de Petri correspond très bien aux besoins d'une étude génétique de la résistance si l'on prend soin de déterminer pour chaque croisement la limite entre plantes résistantes et multiplicatrices en référence aux résultats obtenus avec les parents résistants et multiplicateurs. Une observation stricte des conditions d'utilisation du test en boîtes de Petri et définies par PERSON \& DOUSSINAULT (1978) est cependant nécessaire afin d'éviter toute erreur d'interprétation.

A. Ce travail a révélé la présence de nouveaux gènes de résistance (tabl. 8) dans des cultivars déjà bien étudiés (ANDERSEN, 1975) :

$\mathrm{AB}$ et $\mathrm{C}$ dans « Siri »,

EF ( = NO) et $\mathrm{G}(=\mathrm{K}) \mathrm{H}$ dans «Ortolan »,

$\mathrm{IJ}$ et $\mathrm{G}(=\mathrm{K}) \mathrm{L}$ dans « $\mathrm{P}$ 31-322-1».

Cette révélation de nouveaux gènes n'est pas surprenante en ce qui concerne les pathotypes méridionaux Fr 1 et Fr 2 d' $H$. avenae puisque ces derniers n'ont pas d'équivalents en Europe du Nord où les travaux d'étude génétique de la résistance de l'orge à $H$. avenae ont été réalisés. Par contre, la présence de gènes de résistance tels que $\mathrm{C}$ dans "Siri », $\mathrm{G}(=\mathrm{K}) \mathrm{H}$ dans «Ortolan », G (= K) L dans «P 31-322-1 » vis-à-vis du pathotype Fr 3, similaire au pathotype danois $\mathrm{Ha} 11$

TABLEAU 7

Etude du mode d'hérédité de la résistance de 4 cultivars d'orge au pathotype Fr 3 d'Heterodera avenae en France : analyse de la descendance $F 2$. Inheritance of the resistance of 4 barley varieties to pathotype Fr 3 of Heterodera avenae : study of the F 2 progeny.

\section{Croisement «Siri » $x$ «Ortolan »}

gènes de résistance

Siri = résistant à $\operatorname{Fr} 3 ; \mathrm{AB}(\mathrm{Fr} 2), \mathrm{C}(\operatorname{Fr} 3), \mathrm{Ha} 2=\mathrm{M}=\mathrm{D}(\mathrm{Fr} 4)$ Ortolan $=$ résistant à $\operatorname{Fr} 3 ; \mathrm{EF}=\mathrm{NO}(\mathrm{Fr} 1), \mathrm{GH}(\mathrm{Fr} 3)$

1) 53 plantes F 2

$5 \mathrm{M} 48 \mathrm{R}$

2) $7 / 64 \mathrm{M} 57 / 64 \mathrm{R}$

3) $\mathrm{X}^{2}=0,123$

$0,70<\mathrm{p}<0,80$

4) 3 gènes dominants dont 2 complémentaires, $G$ et $H$, et

1 indépendant, $C$

$\mathrm{C}$ indépendant de $\mathrm{GH}, \mathrm{EF}=\mathrm{NO}$,

$\mathrm{GH}$ indépendant de $\mathrm{AB}, \mathrm{C}, \mathrm{Ha} 2=\mathrm{M}=\mathrm{D}$

Croisement «Ortolan » $\times$ «P 31-322-1 »

gènes de résistance

$\mathrm{P}$ 31-322-1 = résistant à Fr 3 ; IJ (Fr 2), KL (Fr 3), Ha $2=\mathrm{D}=\mathrm{M}$ (Fr 4)

Ortolan $=$ résistant à Fr $3 ; \mathrm{EF}=\mathrm{NO}(\mathrm{Fr} 1), \mathrm{GH}(\mathrm{Fr} 3)$

1) 89 plantes $\mathrm{F} 2$ $5 \mathrm{M} \quad 84 \mathrm{R}$

2) $1 / 16 \mathrm{M} 15 / 16 \mathrm{R}$

3) $\mathrm{X}^{2}=0,0602$ $0,80<\mathrm{p}<0,90$

4) 2 gènes dominants indépendants, $\mathrm{H}$ et $\mathrm{L}$, complémentaires de 2 gènes dominants identiques, $\mathrm{K}$ et $\mathrm{G}$

$\mathrm{G}(=\mathrm{K}) \mathrm{H}$ indépendants de $\mathrm{IJ}$, Ha $2=\mathrm{D}=\mathrm{M}$

$\mathrm{K}(=\mathrm{G}) \mathbf{L}$ indépendants de $\mathrm{EF}=\mathrm{NO}$
Croisement «Vogue » $x$ «Siri »

gènes de résistance

Vogue $=$ résistant à $\mathrm{Fr} 3 ; \mathrm{NO}=\mathrm{EF}(\mathrm{Fr} 1), \mathrm{P}(\mathrm{Fr} 3)$

Siri $=$ résistant à Fr 3 ; $\mathrm{AB}(\mathrm{Fr} 2), \mathrm{C}(\mathrm{Fr} 3), \mathrm{Ha} 2=\mathrm{D}=\mathrm{M}(\mathrm{Fr} 4)$

1) 84 plantes $\mathrm{F} 2$

$4 \mathrm{M} 80 \mathrm{R}$

2) $1 / 16 \mathrm{M} 15 / 16 \mathrm{R}$

3) $\mathrm{X}^{2}=0,317$

$0,50<\mathrm{p}<0,70$

4) 2 gènes dominants indépendants, $C$ et $P$

$\mathrm{P}$ indépendant de $\mathrm{AB}, \mathrm{C}, \mathrm{Ha} 2=\mathrm{D}=\mathrm{M}$

$\mathrm{C}$ indépendant de $\mathrm{NO}=\mathrm{EF}, \mathrm{P}$

Croisement «P 31-322-1» $\times$ «Vogue »

gènes de résistance

P 31-322-1 = résistant à Fr 3, IJ (Fr 2), KL (Fr 3), Ha $2=M=D$ (Fr 4)

Vogue = résistant à $\mathrm{Fr} 3, \mathrm{NO}=\mathrm{EF}(\mathrm{Fr} 1), \mathrm{P}(\mathrm{Fr} 3)$

1) 96 plantes $F 2$ $9 \mathrm{M} \quad 87 \mathrm{R}$

2) $7 / 64 \mathrm{M} 57 / 64 \mathrm{R}$

3) $\mathrm{X}^{2}=0,240$ $0,50<p<0,70$

4) 3 gènes dominants dont 2 complémentaires, KL, et 1 indépendant, $P$

$\mathrm{P}$ indépendant de $\mathrm{IJ}, \mathrm{KL}, \mathrm{Ha} 2=\mathrm{D}=\mathrm{M}$ $\mathrm{KL}$ indépendants de $\mathrm{NO}=\mathrm{EF}, \mathrm{P}$

$\mathrm{R}$ : résistant ; $\mathrm{M}$ : multiplicateur ; 1) résultats ; 2) hypothèse ; 3) test de l'hypothèse ; 4) conclusion. 
TABLEAU 8

Gènes de résistance à l'encontre des pathotypes d'Heterodera avenae. Genes for resistance to the pathotypes of Heterodera avenae.

\begin{tabular}{|c|c|c|c|c|c|c|}
\hline \multirow{2}{*}{$\begin{array}{l}\text { Pathotypes } \\
\text { d'H. avenae }\end{array}$} & \multicolumn{2}{|c|}{ Danois } & \multicolumn{4}{|c|}{ Français } \\
\hline & Ha 11 (A) & $\mathrm{Ha} 12(\mathrm{C})$ & $\mathrm{Fr} 1=\mathrm{Ha} 41$ & Fr 2 & Fr 3 & Fr $4=\mathrm{Ha} 12$ \\
\hline \multicolumn{7}{|l|}{ Variétés d'orge } \\
\hline Siri & $\mathrm{Ha} 2$ & $\mathrm{Ha} 2$ & Pas de résistance & $\begin{array}{c}2 \text { gènes dominants } \\
\text { complémentaires } \mathrm{AB} \\
\text { indépendants de } \\
\mathrm{EF}=\mathrm{NO}, \mathrm{G}(=\mathrm{K}) \mathrm{H}, \mathrm{P}\end{array}$ & $\begin{array}{c}1 \text { gène dominant } \mathrm{C} \\
\text { indépendant de } \\
\mathrm{G}(=\mathrm{K}) \mathrm{H}, \mathrm{EF}=\mathrm{NO}, \mathrm{P}\end{array}$ & $\begin{array}{l}1 \text { gène dominant } \\
\text { Ha } 2\end{array}$ \\
\hline Ortolan & Ha 1 & $\begin{array}{l}\text { Pas de } \\
\text { résistance }\end{array}$ & $\begin{array}{c}2 \text { gènes dominants } \\
\text { complémentaires } \\
\mathrm{EF}=\mathrm{NO} \\
\text { indépendants de } \mathrm{AB}, \mathrm{C} \\
\mathrm{Ha} 2=\mathrm{D}=\mathrm{M}, \mathrm{J}, \\
\mathrm{K}(=\mathrm{G}) \mathrm{L}\end{array}$ & Pas de résistance & $\begin{array}{c}2 \text { gènes dominants } \\
\text { complémentaires } \\
\mathrm{G}(=\mathrm{K}) \mathrm{H} \\
\text { indépendants de } \mathrm{AB}, \mathrm{C} \\
\text { Ha } 2=\mathrm{D}=\mathrm{M}, \mathrm{IJ}\end{array}$ & Pas de résistance \\
\hline P $31-3221$ & $\begin{array}{c}\mathrm{Ha} 1 \text { et } \\
\mathrm{Ha} 2\end{array}$ & $\mathrm{Ha} 2$ & Pas de résistance & $\begin{array}{c}2 \text { gènes dominants } \\
\text { complémentaires } 1 \mathrm{~J} \\
\text { indépendants de } \\
\mathrm{EF}=\mathrm{NO}, \mathrm{G}(=\mathrm{K}) \mathrm{L}, \mathrm{P}\end{array}$ & $\begin{array}{l}2 \text { gènes dominants } \\
\text { complémentaires } \\
K(=G) L \\
\text { indépendants de } \\
E F=N O, P\end{array}$ & $\begin{array}{l}1 \text { gène dominant } \\
\text { Ha } 2\end{array}$ \\
\hline Vogue & - & - & $\begin{array}{c}2 \text { gènes dominants } \\
\text { complémentaires } \mathrm{NO}=\mathrm{EF} \\
\text { indépendants de } \mathrm{AB}, \\
\mathrm{C}, \mathrm{Ha} 2=\mathrm{M}=\mathrm{D}, \mathrm{IJ}, \\
\mathrm{K}(=\mathrm{G}) \mathrm{L}\end{array}$ & Pas de résistance & $\begin{array}{c}1 \text { gène dominant } P \\
\text { indépendant de } A B, \\
C, \mathrm{Ha} 2=\mathrm{D}=\mathrm{M}, \mathrm{IJ}, \\
\mathrm{K}(=\mathrm{G}) \mathrm{L}\end{array}$ & Pas de résistance \\
\hline
\end{tabular}

(A), ne correspond pas aux résultats attendus et cités par ANDERSEN \& ANDERSEN (1975, 1982). L'explication la plus probable est que notre pathotype $\mathrm{Fr} 3$ a en fait une génétique de la virulence différente de celle du pathotype Ha 11 (A).

Les résultats obtenus montrent également que la variété d'orge française, "Vogue ", ayant un comportement identique à celui d'“ Ortolan " vis-à-vis des 4 pathotypes d' $H$. avenae, sa résistance dépend :

- de 2 gènes dominants complémentaires, EF ( = NO), retrouvés dans « Ortolan " à l'égard de Fr 1 ;

- d'un gène dominant non connu, $P$, dans "Ortolan » à l'encontre de Fr 3.

Il nous est clifficile d'identifier tous les nouveaux gènes trouvés à d'autres sources de résistance connues et répertoriées par COOK \& YORK (1982) car, là encore, les travaux étrangers ne sont faits qu'avec des pathotypes identiques à nos 2 pathotypes septentrionaux. Il faudrait de plus réaliser un certain nombre de croisements avec d'autres lignées d'orge résistantes, ce qui représente un travail constituant à lui seul une autre étude, dans laquelle viendrait s'ajouter la recherche d'identité des gènes $\mathrm{AB}$ et $\mathrm{IJ}$.

B. Ce travail montre que, comme le propose ANDERSEN, Fr 4 peut être considéré comme équivalent au pathotype $\mathrm{C}$ et être confondu avec lui sous le nom de Ha 12. Les 2 pathotypes Fr 4 et Fr 2 sont différents puisque les systèmes génétiques mis en jeu pour la résistance sont différents. En effet, 2 gènes dominants complémentaires, $\mathrm{IJ}$ et $\mathrm{AB}$, sont nécessaires pour induire la résistance des orges «P 31-322-1 》 et «Siri » à l'encontre de Fr 2, alors qu'un seul gène dominant suffit pour le pathotype $\mathrm{Fr} 4$. Ces résultats viennent conforter les différences, déjà notées par RIVOAL \& PERSON-DEDRYVER (1982), au niveau soit des gammes d'hôtes parmi les variétés d'avoine, soit de l'agressivité ou capacité du nématode à se multiplier plus ou moins sur orge, blé et avoine.

Il faut également admettre la non-identité des pathotypes $\mathrm{Ha} 11$ (A) et $\mathrm{Fr} 3$, puisque l'analyse génétique des résistances de "Siri », "Ortolan » et «P 31322-1 " différencient les gènes responsables de la virulence de ces 2 pathotypes. Ce résultat est sans doute le plus surprenant car, jusqu'à présent, nous n'avions jamais noté de différences entre ces 2 pathotypes.

Une terminologie différente de $\mathrm{Ha} 11$ et $\mathrm{Ha} 12$ doit donc être donnée aux pathotypes français Fr 3 et Fr 2.

C. L'ensemble de ces résultats concernant pathotypes et cultivars résistants peut constituer une base importante et fiable pour un programme de sélection d'orges résistantes à l'encontre d' $H$. avenae en France. 


\section{RÉFÉRENCES BIBLIOGRAPHIQUES}

Andersen S., 1975. International test assortment for Heterodera avenae and other species. Mimeo. doc., Agric. Univ., Copenhagen, $3 \mathrm{p}$.

Andersen S., Andersen K., 1970. Sources of genes which promote resistance to races of Heterodera avenae Woll. EPPO Public., Ser. $A$, 54, 29-36.

Andersen S., Andersen K., 1982. Suggestions for determination and terminology of pathotypes and genes for resistance in cyst-forming nematodes, especially Heterodera avenae. EPPO Bull., 12, 379-386.

Cook R., York P. A., 1982. Resistance of cereals to Heterodera avenae : methods of investigation, sources and inheritance of resistance. EPPO Bull., 12, 423-434.

Person F., Doussinault G., 1978. Influence de la température et des caractères des races d'Heterodera avenae Woll. sur la validité d'un test en conditions contrôlées, utilisable en sélection des céréales. $A n n$. Amélior. Plant., 28, 513-527.
Rivoal R., 1977. Identification des races biologiques du nématode à kystes des céréales, Heterodera avenae Woll. en France. Ann. Zool. Ecol., Anim., 9, 261-272.

Rivoal R., 1979. Biologie d'Heterodera avenae Wollenweber en France. II. Etude des différences dans les conditions thermiques d'éclosion des races Fr 1 et Fr 4, Rev. Nématol., 2, 233-248.

Rivoal R., Person F., Caubel G., Scotto la Massese C., 1978. Méthodes d'évaluation de la résistance des céréales au développement des nématodes : Ditylenchus dipsaci, Heterodera avenae et Pratylenchus spp. Ann. Amélior. Plant., 28, 31-54.

Rivoal R., Person-Dedryver F., 1982. Caractérisation des pathotypes d'Heterodera avenae en France : influence de la période de culture sur le pouvoir discriminant de cultivars d'Avena sativa et différences dans la capacité à former des femelles. EPPO Bull., 12, 387-391. 